showed a high Calcium of $2.89 \mathrm{mmol} / \mathrm{L}$ with a low urine Calcium supporting the likely diagnosis of familial hypocalciuric hypercalcaemia. Molecular analysis of AP2S1 gene was abnormal. This result confirmed a molecular disgnosis of $\mathrm{FHH}$ type 3.

Discussion $\mathrm{FHH}$ is an autosomal dominant condition. FHH1 is caused by loss of function in CASR gene, FHH2 by a mutation in GNA11 gene and FHH3 identified as mutation in AP2S1 gene. Mutations lead to an elevation of the normal set point for maintaining normal plasma calcium levels. This causes mild to moderate hypercalcaemia with ionised calcium levels usually within $10 \%$ of the upper limit of normal. Urine calcium/creatinine ratio tends to be low in $\mathrm{FHH}$ whereas it is usually elevated in primary hyperparathyroidism. In the classic description of $\mathrm{FHH}$, individuals are asymptomatic with normal bone density and no intervention is usually required.

\section{P41 NOT YOUR TYPICAL RICKETS CASE}

Rachel Beckett*, Emmeline Heffernan. Royal Belfast Hospital for Sick Children, Belfast, UK

\subsection{6/archdischild-2019-epa.396}

Presentation A healthy caucasian 3 year old girl was referred due to bowing of her femora, apparent since she started walking at 13 months. She was reported to be clumsy and tire easily. There was no history of fractures or leg pain. Her height had dropped from the $75^{\text {th }}$ centile to between the $25^{\text {th }}$ and $50^{\text {th }}$ centile. Initial investigations showed mildly low corrected calcium and phosphate, slightly raised alkaline phosphatase and a sufficient vitamin $\mathrm{D}$ level of $50 \mathrm{nmol} / \mathrm{L}$. She was treated with oral Vitamin D supplements. X-ray of knees was normal. On review after 6 months, bowing had progressed and height had fallen further. This led to the consideration of rarer forms of rickets.

Further investigations Urinary calcium creatinine ratio was normal at 0.07 but urinary phosphate: creatinine ratio was elevated at 4.36; with reduced tubular reabsorption of phosphate - in keeping with a diagnosis of $\times$ linked hypophosphataemic rickets. This was confirmed by detection of a mutation in the PHEX gene.

Skeletal survey showed lower limb abnormalities, including flared metaphyses, widening of the growth plates and buttressing of the femur and tibia. Renal ultrasound showed no nephrocalcinosis. Parental blood tests showed a slightly low phosphate level in her mother.

Progress and treatment She was treated with oral phosphate supplements and alfacalcidol. Since commencing treatment her growth has improved and she remains active but still tires easily. She was subsequently diagnosed with moderate sensorineural hearing loss, another feature of hypophosphataemic rickets, and has had bilateral hearing aids fitted.

$\mathrm{X}$ linked hypophosphataemic rickets Rickets is a disorder of the growth plate, due to inadequate supply of phosphate to growing bones. Mutations in the PHEX gene cause increased levels of fibroblast growth factor 23 (FGF23), resulting in reduced absorption of phosphate in the proximal renal tubule. Although rare, it is the most common form of hereditary rickets, and usually presents in the first 2 years of life. Investigations show low serum phosphate and the key feature is significant phosphaturia (calculated by $\mathrm{TmP} / \mathrm{GFR}$ ). Patients are at increased risk of dental complications, enthesopathy, lumbar lordosis and hearing impairment. Phosphate supplements replace renal losses and calcitriol increases phosphate absorption from the gut and reduces PTH, preventing nephrocalcinosis. A new treatment, Burosumab is a monoclonal IgG1 antibody that binds excess FGF23. This normalises phosphate levels and improves bone mineralisation.

\section{P42 SEPTIC ARTHRITIS OF ELBOW JOINT IN AN 11 MONTH BABY FOLLOWING CHICKENPOX, A CASE REPORT}

Mohamed Elrih*, Ali Omer, Mohamed Shalan, Mr William Gaine. Sligo University Hospital, Sligo, Ireland

\subsection{6/archdischild-2019-epa.397}

An 11 month baby girl presented with 1 week history of left elbow pain and swelling with restricted mobility, on and off fever treated with paracetamol at home. 3 weeks prior to her presentation she had chickenpox with generalized skin rashes. Baby has no past medical history and up to date with her vaccinations. Clinically elbow was swollen with redness at the dorsal lateral side mainly, very tender to touch with almost no range of motion at a slight flexed position. Routine bloods showed significant increase in the inflammatory markers, ultra-sound of the elbow show moderate joint effusion with fluid in the anterior and posterior aspects of the joint with small foci of debris within the effusion, Synovial thickening and increased vascularity with no irregularity of the underlying bony contours. As simple aspiration was difficult to be performed given her age, patient was taken to theater and urgent drainage on the day of presentation was done, with about $10-15 \mathrm{ml}$ of yellowish discharge drained from the lateral side of the elbow. Blood culture along with the joint fluid showed growth of Streptococcus Pyogenes which was sensitive to Penicillin. After 4 weeks of Intravenous Penicillin and another 4 weeks of oral Amoxicillin, baby showed complete recovery both clinically and on her routine bloods.

\section{P43 THE POWER OF THE MIND}

${ }^{1}$ Cathleen McCarrick*, ${ }^{1}$ Noelle Cassidy, ${ }^{2}$ Karen Kelleher. ${ }^{1}$ Temple Street, Children's University Hospital, Dublin, Ireland; '2Our Lady's Children's Hospital, Crumlin, Dublin, Ireland

\subsection{6/archdischild-2019-epa.398}

Introduction Somatoform disorders are a challenge for all branches within medicine but particularly for those working within the paediatric sphere. They are characterized by physical symptoms that are inconsistent with or not fully explained by any underlying medical or surgical diagnosis. In order to reach the diagnosis of a somatoform disorder, children are typically subjected to a number of investigations.

Somatoform disorders can have a significant impact within two domains; the economic impact on healthcare resources in reaching a diagnosis and the effect on children in terms of the underlying emotional stress, the practical impact on families, school absenteeism and the treatment of such a condition. For the purposes of this study, we examined the incidence of somatoform disorders within an orthopaedic cohort of patients. 
Methods This was a retrospective cohort study which examined the incidence of orthopaedic admissions that were attributed to a somatoform disorder over a five year period. All cases which were diagnosed as having a somatoform disorder were included. The individual presentation of each case and the factors of each case that were attributable to the development of such a disorder were assessed in terms of their statistical significance. Factors included childhood trauma, bullying and stressors within the home environment.

Further to that, the economic impact of the investigations and treatments initiated prior to a formal diagnosis of a somatoform disorder were assessed in terms of a cost-benefit analysis framework.

Conclusions Somatoform disorders are a common theme throughout the varying subspecialties of paediatric medicine in particular orthopaedic surgery which has regular admissions attributable to this disorder.

Our research demonstrates that the development of a somatoform disorder is typically attributable to a number of contributing factors. In our study population, some of these factors were found to be statistically significant such as bullying. Further to that, the role of social media was highlighted within several individual cases as a potential factor. This burgeoning influence on children and adolescents highlights the importance of undertaking more research in how this can affect children in terms of their emotional and social development.

Finally, in terms of a cost-benefit analysis the economic costs both in the short-term and long-term were illustrated. More investment and development in this area would lead to economic savings in the long-term.

\section{P44 HEART PATHOLOGY IN PATIENTS WITH SYNDROME MAROTO-LAMY}

${ }^{1}$ Nato Vashakmadze*, ${ }^{1}$ Leyla Namazova-Baranova, ${ }^{1}$ Tatiana Privalova, ${ }^{2}$ Anastasia Rykunova, ${ }^{3}$ Natalya Zhurkova, ${ }^{3}$ Elena Komarova. ${ }^{1}$ Pirogov Russian National Research Medical University (RNRMU), Moscow, Russian Federation; ${ }^{2}$ I.M. Sechenov First Moscow State Medical University (Sechenov University), Moscow, Russian Federation; ${ }^{3}$ National Medical Research Center for Children's Health, Moscow, Russian Federation

\subsection{6/archdischild-2019-epa.399}

Background MucopolysaccharidosistypeIV (syndromeMarotoLamy) is a rare lysosomalstorage disorder resulting from a deficiency of arylsulfataseB due to mutations in the gene ARSB and accumulation of dermatansulfate in organs and tissues. Clinical phenotype is very variable. Patients usually have short stature, multiplex dysostosism, facial dysmorphism, corneal clouding, cardiovascular abnormalities and cervical myelopathy.

Methods 117 patient with various types of mucopolysaccharidosisis observed in our clinic, 8 patients with mucopolysaccharidosistype VI. The average age of diagnostic of MPS VI in this group patients was $65.43 \pm 14,64$ months (5 years and 5 months).

Results and discussion We evaluated the level of N-terminal fragment of its prohormone(NT-proBNP) in 30 patients with various types of MPS (23 boys and 7 girls). The reference values for boys was $\leq 62 \mathrm{PG} / \mathrm{ml}$ for girls $-\leq 83 \mathrm{PG} /$ $\mathrm{ml}$, mean level of NT-proBNP- 81,63 $\pm 8,90 \quad(24,53-216,0)$ $\mathrm{PG} / \mathrm{ml}$. the Most frequent, in $66.6 \%$ of cases, high level of NT-proBNP- $\quad 144.7 \pm 42,6 \quad(102,1-187,3) \quad P G / m l$, was identified in children with Maroto- Lamysyndrome. We observed 8 children with MPS VI, all patients had valvularheart disease. Hypertrophic cardiomyopathy was find in 5 children, pulmonary hypertensionwas diagnosed in 3 patients. Most often, heart failure we have found in patients with Maroto-Lamysyndrome. Patients with MPS VI is progressive heart disease, which is the most cause of death. Heart failure diagnostic in the initial stages is very important for adequate therapy of hemodynamics disorder and prevent its further progression of cardiovascular problems. NT-proBNPis cardiac biomarker for heart failure management. NT-proBNPis a one of the most predictors of prognosis HF, it help to identify high-risk patients who need cardiologic monitoring and more effective therapy.

\section{P45 PARAMETERS FOR EARLY DETECTION OF CARDIOVASCULAR DISEASE IN MAINTENANCE DIALYSIS PEDIATRIC PATIENTS}

${ }^{1}$ Lacramioara Eliza Pop*, ${ }^{1}$ Adriana Monica Bungardi, ${ }^{2}$ Bogdan Bulata, ${ }^{2}$ Dan Delean, ${ }^{2}$ Cornel Aldea, ${ }^{3}$ Simona Cainap. 'Second Department of Pediatrics, Clinical Emergency Hospital for Children, Cluj-Napoca, Romania; ${ }^{2}$ Pediatric Nephrology and Dialysis Department, Clinical Emergency Hospital for Children, Cluj-Napoca, Romania; ${ }^{3}$ Second Department of Pediatrics, 'Iuliu Hatieganu' University of Medicine and Pharmacy, ClujNapoca, Romania

\subsection{6/archdischild-2019-epa.400}

Introduction The excessive mortality seen in patients with endstage renal disease (ESRD) is closely related to cardiac disease. We aimed to assess if there were distinctive parameters that can early predict cardiac disfunction in children on maintainance dialysis (MD).

Methods From the ESRD database of our tertiary referral centre (2017-2018), we enrolled all patients on MD. For statistical analysis we used STATA software version 13.

Results Twenty-four patients were on MD: mean age ( \pm SD) $14.6 \pm 4.7$ years, mean duration of MD 3.5 years, $55 \%$ male. The study population was divided into two groups: group-1 consisted of patients with cardiomyopathy and group-2 included patients without cardiac disfunction. The two groups were similar regarding demographic characteristics, cardiovascular risk factors and uremia-related metabolic cardiac risk factors. On univariate analysis, a statistically significant difference was seen in the value of serum albumin $(p=0.049)$ and $s$ wave velocity on tissue doppler imaging (TDI) $(p=0.02)$. No significant difference between the two groups was found for type of dialysis (hemodialysis or peritoneal dialysis), growth delay, laboratory parameters like homocisteine and NT-pro BNP (and a lot other), ECG parameters.

Conclusions Some echocardiographic parameters such as systolic dysfunction assesed by $\mathrm{s}$ wave on TDI are the best tools for early diagnosis of cardiac disfunction. No ECG parameters were found to predict early disfunction. There are also bioumoral factors that can alert the clinician regarding cardiac malfunction such low level of seric albumin. Protocols for regular echocardiographic screening, diagnosis and monitoring of heart disease are recommended in the follow-up of these patients in order to provide a proper management of the disease and prevent complications like cardiac disease which is the main cause of morbidity and mortality in children with end-stage renal disease. 